\title{
EFEITO DA GRANULOMETRIA E DA DOSAGEM DE AMINA NA FLOTAÇÃO DE ITABIRITOS
}

\author{
D. A. PIMENTEL, J. F. C. RUSSO, D. B. MAZZINGHY e H. D. G. TURRER \\ Anglo American - Minério de Ferro \\ danielle.pimentel@angloamerican.com
}

Artigo submetido em novembro/2013 e aceito em julho/2014

DOI: $10.15628 /$ holos.2014.1766

\section{RESUMO}

O escalonamento da etapa de flotação é sempre muito discutido na indústria mineral visto que existem diferentes variáveis que interferem na recuperação em massa e metalúrgica dos materiais concentrados através desta operação unitária. Normalmente são testadas em escala de bancada diversas condições de operação como granulometria, dosagem de reagentes, aeração e $\mathrm{pH}$. Neste trabalho foram realizados vários ensaios de flotação com o objetivo de investigar o efeito da granulometria de alimentação e da dosagem de amina na flotação do itabirito compacto do Projeto Minas-Rio, de propriedade da Anglo American. Os trabalhos foram conduzidos utilizando uma amostra composta de testemunhos de sondagem, incluindo preparação, análise granuloquimica, moagem, estudo de liberação, deslamagem e testes cinéticos de flotação rougher. Foram traçadas curvas de recuperação por tempo e através destas foram determinadas as constantes cinéticas de flotação. Os resultados indicaram que para P80 de $45 \mu \mathrm{m}$ é requerido um tempo prolongado de moagem, há elevada perda de material para a lama, baixo rendimento metalúrgico (entre $33,1 \%$ e $57,6 \%$ ) e o teor de ferro no concentrado não satisfatório (entre 7,3\% e $16,4 \%)$. Nos testes com P80 de 75 e $100 \mu \mathrm{m}$, ocorreu boa recuperação de ferro considerando tempos de moagem de 20 minutos para $\mathrm{P} 80$ de $100 \mu \mathrm{m}$ e 40 minutos para $\mathrm{P} 80$ de $75 \mu \mathrm{m}$

PALAVRAS-CHAVE:. Flotação, dosagem de reagente, distribuição de tamanho de partícula, cinética.

\section{EFFECT OF PARTICLE SIZE RANGE AND AMINE DOSAGE IN THE ITABIRITE FLOTATION}

\begin{abstract}
Flotation scale-up is always much discussed in the mineral industry as there are several variables that affect the mass and metallurgical recovery. Typically, many tests are carried out in different operating ranges such as particle size distribution, dosage reagents, aeration and $\mathrm{pH}$. This paper presents the results of many flotation tests that were carried out in order to investigate the effect of the particle size distribution on feed and amine dosage in flotation of compact itabirite ore from Minas-Rio Project, propriety of AngloAmerican. The studies were conducted using samples of drill holes core, including preparation,
\end{abstract}

chemistry analysis, grinding, desliming, liberation and tests for rougher kinetic flotation determination.

Recovery curves were plotted by time and through these results were determined the kinetics flotation constants. The results indicated that $\mathrm{P} 80$ of $45 \mu \mathrm{m}$ required a long time of grinding, there is a high loss material in the slimes, low metallurgical recovery (between $33.1 \%$ and $57.6 \%$ ) and iron content of the concentrate wasn't satisfactory (between $7.3 \%$ and 16.4\%). In tests with P80 of 75 and $100 \mu \mathrm{m}$ resulted a good iron recovery considering grinding time of 20 minutes to $\mathrm{P} 80$ of $100 \mu \mathrm{m}$ and 40 minutes to $\mathrm{P} 80$ of $75 \mu \mathrm{m}$.

KEYWORDS: Flotation, reagent dosage, particle size distribution, kinetics. 


\section{INTRODUÇÃO}

Grande parte dos depósitos brasileiros de minérios de ferro apresentam porções com alto grau de intemperismo gerando quantidades significativas de partículas minerais finas. Além destes finos naturais, presentes em grandes quantidades nos itabiritos friáveis, também ocorre a geração de partículas finas durante as operações de lavra, bem como, nos processos de cominuição (MAPA, 2006).

Para Hout (1983), a escolha do processo de concentração de minério de ferro a ser aplicado, depende de vários fatores, entre eles, o teor do minério e o teor do concentrado que deseja obter, composição química e mineralógica do minério, malha de liberação, tamanho de grãos, fatores econômicos e efeitos sobre a pelotização.

Lopes (2009) descreve que o processo de flotação de minério de ferro tem sido utilizado para: a) concentração de minérios de ferro não magnéticos pela flotação da sílica (flotação reversa) ou da hematita (flotação direta), b) remoção da sílica abaixo de 0,044 $\mathrm{mm}$ dos concentrados magnéticos, c) preparação de superconcentrados para redução direta e d) redução do nível de impurezas, além da sílica, como o sódio, o fósforo e o enxofre.

O processo de flotação consiste da separação físico-química baseado nas diferenças de características de superfície dos minerais. A teoria de flotação é complexa envolvendo três fases: sólidos, água e espuma, e com muitos subprocessos e interações, os quais não são completamente compreendidos (PERES e ARAUJO, 2006; WILLS e NAPIER-MUNN, 2006).

Os minerais representam a fase sólida, com superfície polar ou apolar. A água é a fase líquida, cujas moléculas apresentam um dipolo permanente, portanto, de natureza polar. $\mathrm{E}$ a fase gasosa, o ar, é composta basicamente por moléculas apolares (BALTAR, 2008).

O escalonamento da etapa de flotação é sempre muito discutido na indústria mineral, visto que existem várias variáveis que interferem na recuperação em massa e metalúrgica dos materiais concentrados através desta operação unitária. Segundo Fuerstenau e Somasundaran (2003), considerando que cada minério tem as suas peculiaridades, torna-se essencial que a aplicação industrial da flotação seja antecedida de um estudo em laboratório, para definir a influência das diversas variáveis no processo.

Normalmente, são testadas em escala de bancada, diversas condições de operação, como dosagem de reagentes, granulometria de alimentação, aeração e pH. Os resultados são confirmados através de testes em escala piloto, onde são testados diferentes configurações das etapas rougher, cleaner e scavenger, por exemplo.

O Projeto Minas-Rio, de propriedade da Anglo American, está em fase de implantação, na cidade de Conceição do Mato Dentro - MG, e prevê o beneficiamento de minérios itabiríticos. As etapas do circuito de beneficiamento deste projeto compreende a britagem primária e secundária, prensa de rolos, moagem primária em moinho de bolas, deslamagem, flotação em tanques, moagem secundária em moinho vertical, espessamento, disposição de rejeitos, mineroduto e filtragem. O pellet feed produzido será escoado até o Porto de Açu, na cidade de São João da Barra - RJ, por um mineroduto de, aproximadamente, $525 \mathrm{~km}$ (RIBEIRO et al., 2010). 
Neste trabalho foram realizados vários ensaios de flotação com o objetivo de investigar o efeito da granulometria de alimentação e da dosagem de amina na flotação do itabirito compacto da Serra do Sapo. Os trabalhos foram conduzidos utilizando uma amostra composta de testemunhos de sondagem, incluindo preparação, analise granuloquimica, curva de moagem, estudo de liberação, moagem, deslamagem e testes cinéticos de flotação rougher. Foram traçadas curvas de recuperação por tempo e através destas foram determinadas as constantes cinéticas de flotação.

\section{MATERIAIS E MÉTODOS}

Os testes foram conduzidos utilizando uma amostra composta de testemunhos de sondagem conforme está descrito na figura 1.

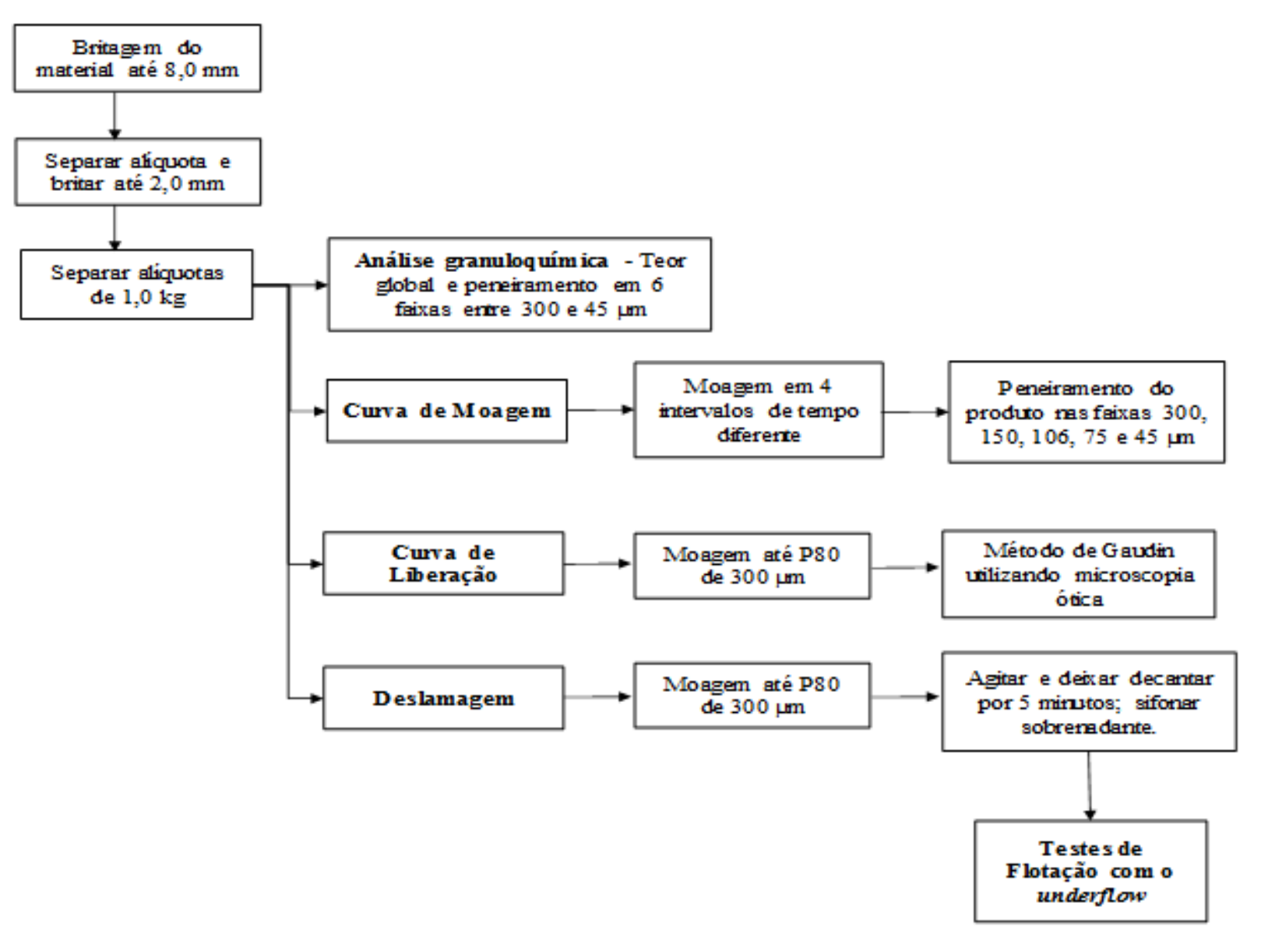

Figura 1: Roteiro de execução dos testes de flotação.

A. Preparação: britagem estagiada de $200 \mathrm{~kg}$ até $8 \mathrm{~mm}$, seguida de homogeneização e quarteamento. Deste material, metade foi separado e reservado. Os $100 \mathrm{~kg}$ restantes foram submetidos à britagem em $2 \mathrm{~mm}$. Por fim, foi utilizado um divisor rotativo para gerar 100 alíquotas de $1 \mathrm{~kg}$.

B. Análise granuloquimica da amostra britada: quarteamento de uma alíquota em 250 e 750 g. Primeiro, $250 \mathrm{~g}$ do material foi submetida à cominuição em moinho de 
panelas construído por anéis de carbeto e tungstênio para determinação do teor global em triplicata. E, em seguida, foi realizado o peneiramento de $750 \mathrm{~g}$ do material em seis faixas entre 300 a $45 \mu \mathrm{m}$, e por fim, o material retido em cada faixa foi submetido à análise química.

C. Curva de moagem: moagem de $2 \mathrm{~kg}$ de minério em quatro intervalos de tempo distintos, seguido de peneiramento do produto nas malhas de 300, 150, 106, 75 e $45 \mu \mathrm{m}$, cálculo da percentagem de massa passante nestas malhas, e construção dos gráficos de tempo de moagem versus $\mathrm{P}_{80}$ e \%passante acumulado versus tempo.

D. Determinação do grau de liberação: moagem de $2 \mathrm{~kg}$ de material em $\mathrm{P}_{80}$ de $30 \mu \mathrm{m}$, seguido de peneiramento nas malhas de 150, 106, 75 e $45 \mu \mathrm{m}$. Foram feitas seções polidas com o material retido em cada peneira, e aplicação do método de Gaudin utilizando microscopia ótica.

E. Deslamagem: foi ajustado o $\mathrm{pH}$ do produto de moagem para 10.5 , seguido de agitação da polpa, e colocada para decantar por 5 minutos. Sifonamento do sobrenadante.

F. Flotação: foi ajustado o pH do underflow da deslamagem para 10.5 , seguida de adição de amido, $500 \mathrm{~g} / \mathrm{t}$, adição de amina na dosagem especificada para o teste. Durante a flotação, a espuma foi recolhida após 1, 2 e 4 minutos, e continuou-se o ensaio ate o esgotamento. Secagem e pesagem dos produtos, e retirada das alíquotas para análise química.

A moagem do material foi realizada em moinho de bolas de laboratório com 0,203 $\mathrm{m}$ de diâmetro por 0,254 m de comprimento, utilizando-se uma carga de $10 \mathrm{~kg}$ de bolas e de $2 \mathrm{~kg}$ de minério, percentagem de sólidos de 65 \% e velocidade de rotação de 67 rpm.

As análises químicas foram realizadas pelo método da fluorescência de raios- $X$ e perda por calcinação (PPC) a $1.000^{\circ} \mathrm{C}$.

Foi testado o efeito combinado dos seguintes fatores na flotação rougher:

- $\mathrm{P}_{80}$ de moagem variando de 45 a $120 \mu \mathrm{m}$;

- Dosagem de amina variando entre 50 e 180 ppm.

As demais condições da flotação foram mantidas fixas nos seguintes valores:

- Percentagem de sólidos da polpa em 30 \%;

- Dosagem de amido de 500 ppm;

- $\mathrm{pH} 10,5$;

- Velocidade do rotor de $900 \mathrm{rpm}$;

- Vazão de ar de 2 L/min;

- Intervalo entre as raspagens da espuma de 5 segundos.

\section{RESULTADOS E DISCUSSÃO}

A Tabela I apresenta a análise granuloquímica do material britado em 2,0 mm e indica teor global de ferro de $30,3 \%$ e sílica de $54,9 \%$. Observa-se que na faixa de $-300+106 \mu \mathrm{m}$ o teor de sílica se aproxima dos $80 \%$. 
Tabela I: Análise granuloquímica do material britado em 2,0 $\mathrm{mm}$.

\begin{tabular}{cccccccc}
\hline Malha $[\mu \mathrm{m}]$ & \% massa & $\mathrm{Fe}$ & $\mathrm{SiO}_{2}$ & $\mathrm{Al}_{2} \mathrm{O}_{3}$ & $\mathrm{P}$ & $\mathrm{Ti}$ & $\mathrm{PPC}$ \\
\hline 300 & 32,1 & 34,7 & 49,3 & 0,5 & 0,032 & 0,06 & 0,2 \\
\hline 150 & 12,0 & 15,9 & 77,6 & 0,2 & 0,014 & 0,03 & 0,0 \\
\hline 106 & 13,6 & 13,2 & 79,9 & 0,2 & 0,010 & 0,03 & 0,0 \\
\hline 75 & 10,1 & 20,4 & 69,1 & 0,3 & 0,013 & 0,04 & 0,0 \\
\hline 45 & 10,1 & 33,9 & 51,2 & 0,5 & 0,018 & 0,04 & 0,1 \\
\hline Fundo & 22,1 & 46,0 & 29,8 & 1,3 & 0,040 & 0,06 & 0,6 \\
\hline Global calculado & 100,0 & 30,5 & 54,8 & 0,6 & 0,025 & 0,04 & 0,2 \\
\hline
\end{tabular}

Em relação ao teor dos demais contaminantes, este é comparativamente baixo para minérios procedentes da região do quadrilátero ferrífero em Minas Gerais.

A figura 2 apresenta as curvas de moagem. A curva da esquerda diz respeito ao tempo de moagem para atingir o $\mathrm{P}_{80}$ pré-definido para a execução dos testes, e a curva da direita trata da percentagem de massa passante acumulada versus tempo.


Figura 2: Tempo de moagem versus P80 (esquerda) e \% Passa. Acum. versus tempo (direita).

Em relação ao grau de liberação de óxidos de ferro, quanto menor o $\mathrm{P}_{80}$, maior é o grau de liberação, no entanto, para menores faixas granulométricas de $\mathrm{P}_{80}$, há maior geração de lama no caso de um circuito de deslamagem (Figura 3 e Tabela 2).

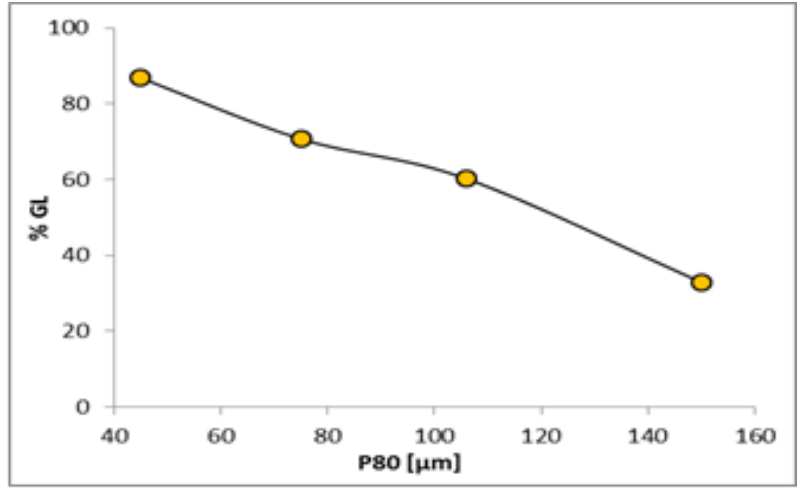

Figura 3: Grau de liberação de óxido de ferro. 
Tabela II - \% lama conforme P80.

\begin{tabular}{ccccc}
\hline P80 & Alim. [g] & OF [g] & UF [g] & \% lama \\
\hline 120 & 18.000 & 643 & 17.357 & $3,6 \%$ \\
\hline 100 & 4.000 & 209 & 3.791 & $5,2 \%$ \\
\hline 75 & 6.000 & 457 & 5.543 & $7,6 \%$ \\
\hline 45 & 4.000 & 1.069 & 2.931 & $26,7 \%$ \\
\hline
\end{tabular}

Conforme descrito na figura 2, para atingir $\mathrm{P}_{80}$ de moagem de $45 \mu \mathrm{m}$ é requerido um tempo prolongado de moagem, 120 minutos na escala de laboratório, além deste tempo prolongado de moagem, há elevada perda de material para a lama (tabela II) e baixo rendimento metalúrgico, em que há recuperação de ferro entre $33,1 \%$ e 57,6 \% e teor do concentrado entre 7,3 \% e 16,4 \% (figuras 5 e 6). Neste sentido, com estes resultados, é desaconselhável a aplicação industrial da moagem em $45 \mu \mathrm{m}$.



Dosagem de amina $=50 \mathrm{ppm}$



Dosagem de amina $=100 \mathrm{ppm}$

Figura 5: \% Recuperação de Fe versus \% Fe no afundado.



Dosagem de amina $=50 \mathrm{ppm}$

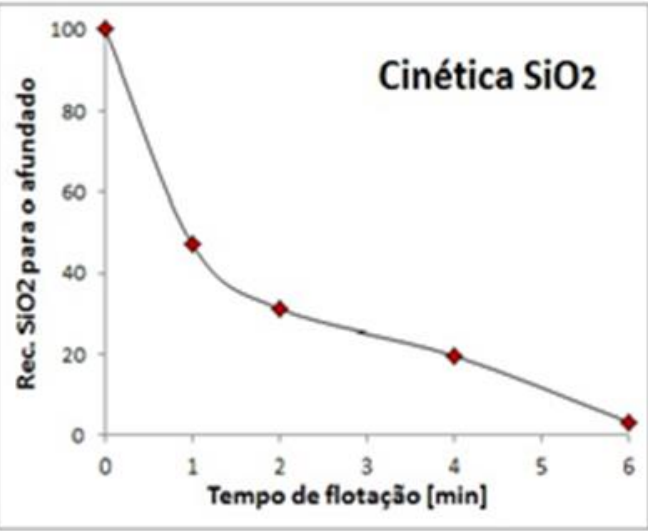

Dosagem de amina $=100 \mathrm{ppm}$

Figura 6: Tempo de flotação versus Recuperação de SiO2 no concentrado.

A utilização de $\mathrm{P}_{80}$ de moagem na faixa de 100 e $75 \mu \mathrm{m}$ se mostrou a mais promissora das opções que foram investigadas. Dentre os vários testes executados, foram selecionados os dois com desempenho mais satisfatório, quais sejam: 
I. $P_{80}$ de $75 \mu \mathrm{m}$, com dosagem de amina de $70 \mathrm{~g} / \mathrm{t}$, o que resultou em uma recuperação de ferro de $57,5 \%$ e teor de $65,3 \%$ no concentrado rougher (Figura 7 ).
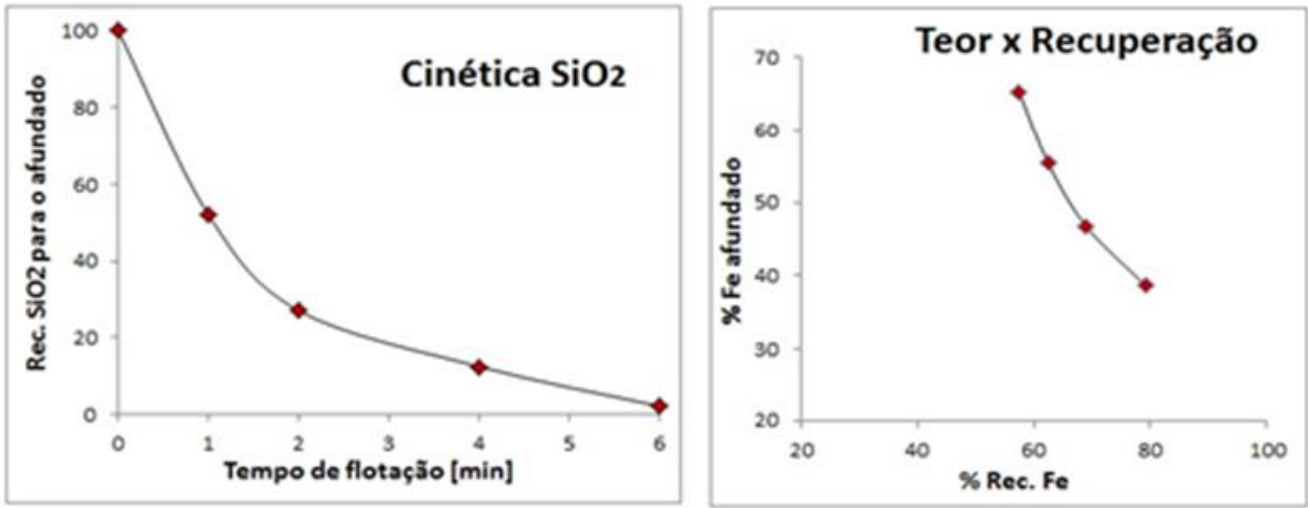

Figura 7: $\mathrm{P}_{80}$ de $75 \mu \mathrm{m}$ - Recuperação de $\mathrm{SiO}_{2}$ no concentrado e recuperação de ferro.

II. $P_{80}$ de $100 \mu \mathrm{m}$, com dosagem de amina de $150 \mathrm{~g} / \mathrm{t}$, resultando em recuperação de ferro de $55,4 \%$ e teor de $63,0 \%$ (Figura 8 a e 8 b).




A figura 9 sumariza as curvas de teor de Fe versus \% recuperação Fe para os testes com $\mathrm{P}_{80}$ de $100 \mu \mathrm{m}$, variando a dosagem de amina em 50, 70 e 100 ppm, e para $\mathrm{P}_{80}$ de $75 \mu \mathrm{m}$, variando a dosagem de amina em 50, 70, 100, 120, 150 e 180 ppm.



Figura 9: Teor de Fe versus \% recuperação Fe, para $\mathrm{P}_{80}$ de 75 e $100 \mu \mathrm{m}$. 


\section{CONCLUSÕES}

Para $\mathrm{P}_{80}$ de $45 \mu \mathrm{m}$ é requerido um tempo prolongado de moagem, 120 minutos na escala de laboratório, e há elevada perda de material para a lama. Somado a isso, há baixo rendimento metalúrgico (recuperação de ferro entre 33,1 \% e 57,6 \%), e o teor de $\mathrm{SiO}_{2}$ no concentrado não é satisfatório (entre $7,3 \%$ e 16,4 \%). Sendo assim, não é aconselhável à aplicação industrial da moagem em $45 \mu \mathrm{m}$.

Quanto aos testes com P80 de 75 e $100 \mu \mathrm{m}$, conforme observado na figura 9, apesar da recuperação ligeiramente inferior, a melhor opção para aplicação industrial é, provavelmente, a utilização de P80 de $100 \mu \mathrm{m}$, em virtude do menor tempo de moagem demandado no laboratório, 20 minutos para P80 de $100 \mu \mathrm{m}$ e 40 minutos para P80 de $75 \mu \mathrm{m}$.

No caso de não se obter um concentrado satisfatório, uma alternativa seria dividir a alimentação do circuito de flotação entre flotação de grossos e flotação de finos, ou seja, adotar o circuito de flotação fracionada. Nesse caso, a alimentação da flotação de grossos teria um intervalo granulométrico em $-0,15+0,045 \mathrm{~mm}$, seguido de uma remoagem, para o caso de um concentrado ligeiramente abaixo do esperado, e por fim, uma flotação de limpeza. E para a flotação de finos, a granulometria da alimentação estaria na faixa $-0,045+0,010 \mathrm{~mm}$, considerando que nesta faixa granulométrica já se espera obter um concentrado final satisfatório na faixa de 67-68\%.

\section{REFERÊNCIAS}

1. BALTAR, C. A. M. Flotação no Tratamento de Minérios. Recife: Departamento de Engenharia de Minas - UFPE, 213p. 2008.

2. FUERSTENAU, M. C., SOMASUNDARAN, P. Flotation. In: FUERSTENAU, M. C. E. Han, K. N. (Ed.). Principles of mineral processing, SME, p.245-306, 2003.

3. HOUOT, R. Beneficiation of iron ore by flotation - Review of industrial and potential applications, International Journal of Mineral Processing, Volume 10, Issue 3, p.183-204, 1983.

4. LOPES, GILMARA MENDONÇA E LIMA, ROSA MALENA FERNANDES. Flotação direta de minério de ferro com oleato de sódio. Rem: Revista Escola de Minas, vol.62, n.3, pp. 323-329, 2009.

5. MAPA, P. S. Rota de processo para o underflow da deslamagem de minério de ferro do concentrador da Samarco Mineração. Dissertação de Mestrado em Engenharia Metalúrgica e de Minas. Escola de Engenharia da UFMG, Belo Horizonte - Minas Gerais, 172 p., 2006.

6. PERES, A. E. C. e ARAUJO, A. C.. A flotação como Operação Unitária no Tratamento de Minérios. In: CHAVES, Arthur Pinto (Organizador). Teoria e Prática do Tratamento de Minérios: Flotação - O Estado da Arte no Brasil. São Paulo: Signus Editora, pp.01-29, 2006.

7. RIBEIRO, Farley Santos; RUSSO, José Francisco Cabello e COSTA, Thiago. Aplicação de prensas de rolos em minério de ferro. Rem: Rev. Esc. Minas, vol.63, n.2, pp. 399-404, 2010.

8. WILLS, B.A., NAPIER-MUNN, T. J.. Mineral Processing technology: An Introduction to the Practical Aspects of Ore Treatment and Mineral Recovery. Oxford: Butterworth-Heinemann, pp.267 - 352, 2007. 